\title{
La ciencia escolar en Perú y Portugal ante los resultados de PISA
}

\author{
School science in Perú and Portugal before the results of PISA
}

\author{
Osbaldo Turpo Gebera 1,2 \\ Escuela Académico Profesional de Derecho, Universidad Continental, Huancayo, Perú \\ ${ }^{2}$ Escuela Académico Profesional de Ciencias y Tecnologías de la Comunicación, Universidad Continental, Huancayo, Perú
}

\section{RESUMEN}

El análisis curricular de los contenidos que comprenden la enseñanza de la ciencia escolar en Perú y Portugal, revelan algunas explicaciones sobre los contrastes de resultados de la evaluación PISA 2012. Unas secuelas vigentes en las pruebas sucesivas, dada la persistencia de las diferencias, y el escaso interés por revertir la situación en Perú, mientras que Portugal experimenta un mayor avance. En esa intención, el análisis curricular, a través de la revisión comparada de sus componentes, muestra la diferencia de concepciones sobre la competencia científica, mientras que Perú enfatiza en la integración de contenidos, a partir de una única docencia; Portugal prioriza la interdisciplinariedad y la co-docencia. También son apreciables las diferencias en la disposición de los conocimientos desarrollados en la educación básica. En torno a los elementos curriculares previstos por PISA, en Portugal predomina la explicación científica $y$, en Perú, la identificación de las cuestiones científicas, dicha capacidad resulta ser la más relevante, y escasamente considerada en Perú. En relación con el conocimiento, en Portugal prevalecen los sistemas físicos y de la tierra y del espacio, por sobre la tecnología en Perú. Sobre las actitudes, éstas, difieren tenuemente. La organización curricular hace apreciables las diferencias en los tiempos curriculares, en Portugal, destinan más periodos que Perú, lo que revelaría un mayor interés por las ciencias. Los factores diferenciales parecen establecer mejores resultados en Portugal que en Perú.

Palabras clave: investigación curricular, educación básica, competencia científica, diseño curricular, PISA

\begin{abstract}
The curricular analysis of the contents that comprise the teaching of school science in Peru and Portugal, reveal some explanations about the contrasts of the results of the PISA 2012 evaluation. Current sequels in the successive tests, the persistence of the differences, and the little interest in reversing the situation in Peru, while Portugal is experiencing greater progress. In this intention, the curricular analysis, through the comparative review of the components, shows the difference of the conceptions about the scientific competence, while Peru emphasizes in the integration of contents, from a single teaching; Portugal prioritizes interdisciplinarity and co-teaching. The differences in the provision of requirements in basic education are also appreciable. Around the curricular elements are provided by PISA, in Portugal, scientific evaluation and, in Peru, the identification of scientific issues, the resulting capacity is the most relevant, and barely considered in Peru. In relation to knowledge, in Portugal physical systems prevail and land and space, by technology in Peru. On attitudes, together, they differ tenuously. The curricular organization makes appreciable differences in curricular times, in Portugal, allocate more periods to Peru, which reveals a greater interest in science. The differential factors for the best results in Portugal than in Peru.
\end{abstract}

Keywords: curricular research, basic education, scientific competence, curricular design, PISA.

Historial del artículo:

Recibido, 22 de febrero de 2017; aceptado, 3 de marzo de 2017; disponible en línea, 15 de mayo de 2017

* Docente investigador en la Universidad Continental.

Correo: oturpo@continental.edu.pe 


\section{INTRODUCCIÓN}

La innovación científico-tecnológica como factor de competitividad demanda habilidades para aprender a aprender, a partir del uso del conocimiento. Afrontar tales retos, exige un conjunto de competencias de orden superior: "aprender cómo aprender" (Hargreaves, 2005). Un desafío que conlleva a la autorregulación, a fin de orientar los procesos del proyecto personal, social o profesional (Yus, Fernández, Gallardo, Barquín, Sepúlveda y Serván, 2013) hacia la formación permanente.

En esa línea, el enfoque de competencias asumido por PISA (Programme for International Student Assessment), responde pragmáticamente a "la resolución de problemas concretos, [y orientados] hacia una mayor utilidad y pertinencia de los contenidos escolares" (Valladares, 2011 , p. 159). Para la Organización para la Cooperación y el Desarrollo Económico (OCDE), se requiere establecer unas competencias individuales relevantes para alcanzar una vida exitosa y un adecuado funcionamiento social (Rychen y Salganik, 2003). PISA, en ese sentido plantea un conjunto de referenciales del logro estudiantil (Rychen y Salganik, 2000), a evidenciarse al final de la escolaridad obligatoria, es decir, un dominio de los conocimientos y habilidades necesarias para actuar con éxito y ser plenamente participativos en la cambiante sociedad (OCDE, 2006, OCDE, 2005). En tal intención, la competencia científica, junto a otras, es evaluada periódicamente, para que los países puedan controlar su desempeño y estimar las metas educativas alcanzadas (OCDE, 2009).

En este estudio, se considera que a pesar de las distancias sociales, económicas y políticas que distingue a los países, es factible, comparativamente, la aproximación desde parámetros compartidos, como el currículo, y más aún, contrastada con los referentes curriculares de PISA. De esa manera, se evidencia cercanías o distancias de los referenciales instituidos. Revela también, el recogimiento de metas y aspiraciones nacionales, al "'conocer' algo que 'veamos' diferente y se adviertan decisiones basadas únicamente en la evidencia local, y que probablemente lleven a políticas fallidas (Baker, 2011). O, de resaltar objetivos ideológicos presentes, en mayor o menor medida, desde otras visiones, y abordados de sobremanera en los currículos. En ese sentido, al comparar los currículos escolares emerge el carácter educativo nacional, expresamente, los fines que ayudan a construir una sociedad basada en la ciudadanía científica y democrática, sobre la base de la diversidad y los derechos humanos (Benavot \& Truong, 2008). Tales aspiraciones, comportan implementar una educación con "un nivel razonable de calidad, como [...] componente y causa principal del desarrollo social y económico futuro de una nación" (Baker, 201 1, p. 91). Aporta a su conocimiento, pensar sobre las desigualdades educativas, desde las evaluaciones, y la reforma de los sistemas educativos (Baker \& LeTendre, 2005).

A fin de determinar la contribución de la educación en ciencias o ciencia escolar, se aborda curricularmente la enseñanza de la competencia científica en Perú y Portugal. Se analiza los presupuestos educativos que explican los contrastes de logro en la prueba PISA 2012. Propiamente, se evalúa la correspondencia de los diseños curriculares con dichos resultados (Gil y Martínez, 2005), en dos realidades contrapuestas. Partimos del supuesto de que los currículos responden a prácticas culturales contextualizadas e históricas, constituidas de habilidades, conocimientos, actitudes y formas de comportamiento. Visto así, la comparación de los currículos de la competencia científica proporciona evidencias para reconocer las diferencias de resultado.

Tabla 1

Indicadores de desarrollo socioeconómico y educativo en Perú y Portugal.

\begin{tabular}{lrcrc}
\hline \multirow{2}{*}{ Indicadores } & \multicolumn{2}{c}{ Perú } & \multicolumn{2}{c}{ Portugal } \\
\cline { 2 - 5 } & Puntaje & Posición & Puntaje & Posición \\
\hline Índice de Desarrollo Humano 2013 & 0,741 & & 0,816 & \\
Esperanza de vida al nacer & 74,200 & Alto/ & 79,700 & Muy Alto/ \\
Años promedio de escolaridad & 8,700 & $77^{\circ}$ & 7,700 & $43^{\circ}$ \\
Años esperados de escolaridad & 13,200 & & 12,000 & \\
Índice de Desarrollo Educativo & 0,925 & & 0,969 & \\
Índice de la EPT relativo al género & 0,960 & Medio/ & 0,949 & Alto/ \\
Tasa de supervivencia en 5 de primaria & 0,872 & $72^{\circ}$ & 0,990 & $47^{\circ}$ \\
Gasto público en educación (\% del PIB) & 2,800 & & 5,600 & \\
Proporción alumnos/maestro (Primaria) & 20,000 & & 11,000 & \\
Coeficiente de Gini & 0,481 & $129^{\circ}$ & 0,345 & $56^{\circ}$ \\
\hline
\end{tabular}




\section{Contextos de referencia}

Los currículos de Perú y Portugal responden a sociedades dispares. Por un lado, Perú asentado en una incipiente modernización, aun desequilibrada y en crecimiento económico. En el otro, Portugal, en renovación acelerada, aunque rezagado e inmerso en una aguda crisis económica (Turpo, 2014). Las diferencias muestran las complejas relaciones con los centros de poder. Aunque con particularidades y luego de recorrer por sistemas autoritarios, prosperan hacia la consolidación democrática y a una intensa institucionalización. En Perú, la dinámica se presenta precipitada, por la "creciente influencia de los organismos internacionales sobre la escolarización y educación" (Pereyra, Kotthoff y Cowen, 2013, p. 8).

La tabla muestra no solo las distancias socioeducativas que los separa, sino también las dinámicas socioeconómicas subyacentes. Entre los indicadores educativos, Perú tiene mayores índices que Portugal, en años promedio y esperados de escolaridad. Superioridad que no necesariamente revela mejores logros de aprendizaje, pues Perú mejora en la cobertura del servicio, más no en la calidad de la educación, dada la inequidad y los déficits de calidad presentes (Espinoza y Torreblanca, 2003), los mismos que no aseguran "a la gran mayoría de estudiantes un logro académico que supere niveles magros en las tres competencias evaluadas" (Unidad de Medición de la Calidad, 2014, p. 79). Si bien Perú, presenta avances educativos, requiere de más impulsos para reducir las "desigualdades horizontales entre las poblaciones indígenas y las de ascendencia europea" (Programa de las Naciones Unidas para el Desarrollo, 2013, p. 65).

El intervencionismo de la OCDE, Banco Mundial, etc., está en la actualidad, favoreciendo la transición hacia un único "horizonte cognitivo", con similares fronteras ideológicas y territoriales, a través de discursos persuasivos y extensibles al mundo globalizado. Una dinámica impuesta como instrumento de gobernanza educativa para la normalización y homogenización de espacios comunes de conocimiento, y la consiguiente, institucionalización de un poder supranacional (Pereyra et al., 2013, Lawn, 2011, Meyer \& Benavot, 2013).

La atención del currículo en la evaluación del aprendizaje ha adquirido en las últimas décadas una importancia singular (OECD, 2004). Los organismos internacionales asumen a las evaluaciones como medio para comparar los distintos sistemas educativos, confrontando los aprendizajes en áreas claves, como matemáticas, ciencias y comunicación. De esa manera, esperan favorecer la calidad y equidad educativa.

La eficiencia del sistema educativo, sin duda, discurre por la evaluación del aprendizaje, y aporta a situar la calidad en el centro de las reformas educativas $y$, esencialmente, de las reformas curriculares. Las evaluaciones como PISA hacen emergente la imperiosa necesidad de cuestionar el sentido del currículo, de reorientar la actualización curricular y mejorar la eficacia y calidad educativa, mediante redireccionamientos de recursos para la educación (Leibfried, 2009). Sugieren Coll y Martin (2006), que alinear los currículos a estándares educativos, con el pretexto de obtener mejores resultados en función a los rankings académicos, refuerza la disociación entre evaluaciones de rendimiento y procesos de cambio curricular. En todo caso, se corre "el riesgo de otorgar subrepticia y erróneamente al esquema una capacidad que por sí solo no tiene ni puede tener: la de generar y legitimar las intenciones educativas que marcan la orientación y los contenidos de los cambios curriculares" (Coll y Martin, 2006, p. 2223). Por consiguiente, abordar de forma comparada los currículos, contribuye a entender las estructuras subyacentes a los sistemas nacionales (Baker \& LeTendre, 2005), y a reconocer las "agendas idénticas" de reforma educativa (Jones, 2007).

En tales decisiones, la OCDE, asesora y elabora recomendaciones de política educativa para sus países miembros e, incluso para los países asociados. Avanzan así, hacia un proceso de modulación educacional regido por una nueva gobernanza mundial, mantenida en una estandarización de exámenes a gran escala, como instrumento principal de control educativo (Tröler, 2009). Las nuevas perspectivas culturales y educativas se manifiestan en los currículos, mediante alineamientos impulsados por referentes, como PISA. Convenientemente, la globalización incide en cambios curriculares que se contraponen al énfasis en lo nacional, así como en la preparación de individuos dentro del enfoque de derechos y de autonomía para participar en la sociedad nacional y global, al mismo tiempo. Tales influencias "produce[n] y transmite[n] doctrinas universales acerca de los derechos, capacidades y habilidades de los individuos, sobre la importancia de la igualdad individual y colectiva, y acerca del valor de las ciencias y la razón" (Astiz, 2014 , p. 4), a través de currículos globales, altamente institucionalizados y distantes de las realidades locales. PISA distinguen determinadas "capacidades, habilidades y aptitudes que, en conjunto, permiten a la persona resolver problemas y situaciones de la vida" (OCDE, 2009, p. 7). Simbólicamente, encarnan la potencialidad del uso de recursos individuales (habilidades, actitudes, conocimientos y experiencias) para afrontar las tareas en un contexto definido. Pedagógicamente, los desempeños "visibilizan" a las competencias, revelando la "finalidad de la formación y no del conocimiento descontextualizado" (Jonnaert, Barrette, Masciotra y Yaya, 2008, p. 25). 


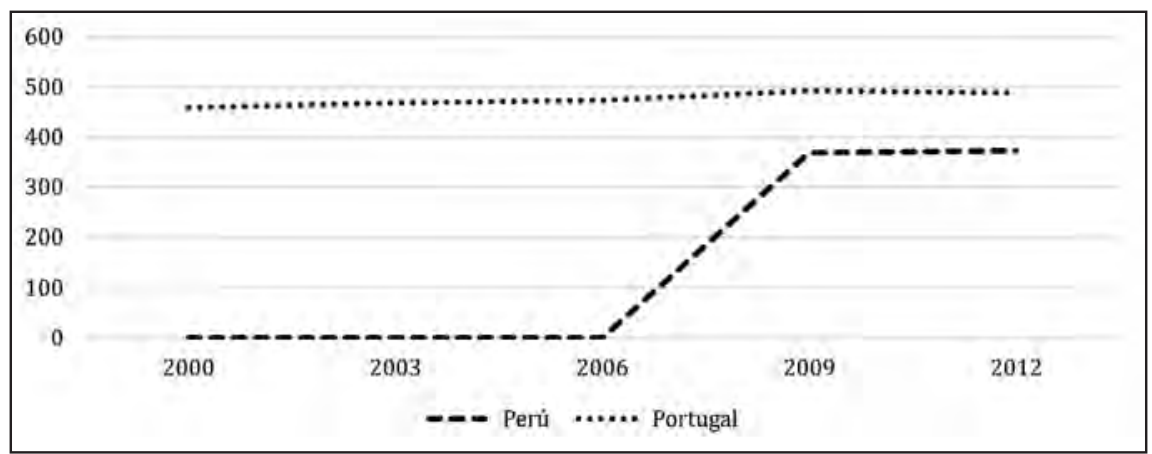

Figura 1:Perú vs. Portugal; Evolución del logro de la competencia científica, según la prueba PISA.

Fuente: Elaborado en base a informes de la OCDE (Turpo, 2014).

\section{PISA y la competencia científica}

La competencia científica, según PISA, "incluye los conocimientos científicos y el uso que haga de esos conocimientos haga un individuo para identificar preguntas, adquirir nuevos conocimientos, explicar los fenómenos científicos y sacar conclusiones basadas en evidencias, sobre asuntos relacionados con la ciencia" (OCDE, 2006, p. 17). Alcanzar esas capacidades implica un dominio científico. En esa línea, PISA evalúa la "comprensión del proceso de investigación científica" (OCDE, 2009, p. 18), a través de la combinación de acciones dirigidas y enmarcadas en un escenario concreto y significativo para resolver problemas o alcanzar algún objetivo.

En la tabla 2, las diferencias de resultado de la competencia científica muestran a Perú, como país invitado, superado ampliamente, por Portugal, país miembro de la OCDE. Este último, decrece en el 2012 $y$, el primero, avanza tenuemente. Ambos, mantienen distancias respecto a los logros de la competencia científica. Asumiendo los desempeños de los países de la OCDE (promedio de 501), Portugal se ubicaría entre los lugares $22^{\circ}$ a $27^{\circ}$, a 12 puntos del promedio y 8 del global (Ministério da Educação e Ciência, 2014). Contrariamente, Perú, aunque acrecentó en algo más de $1 \%$ (considerable para quien viene de logros mínimos), aún está muy distante de la media (128 puntos) y del promedio global (124 puntos). Tales resultados lo ubican en el último lugar del ranking PISA 2012.

Perú, para progresar hacia resultados más satisfactorios, debe acelerar su ritmo de ascenso, para resurgir. Según PISA, cada 38 puntos de logro en ciencias corresponden a un año de escolaridad de la OCDE (OCDE, 2009). Visto así, dista 3,4 y 3 años de escolaridad de la OCDE y de Portugal,

\begin{tabular}{l}
\hline Principales resultados de desempeño en ciencias \\
\hline \% de estudiantes con mayor rendimiento \\
\hline \% de estudiantes con menor rendimiento vs. Niñas \\
\hline Resiliencia \\
Leyenda
\end{tabular}

Figura 2:Desempeño en el rendimiento y entorno social de la competencia científica. Fuente: : Adaptado de OCDE (2012). 
respectivamente. En Perú, un 69\% de estudiantes esta en los más bajos niveles de logro en ciencias (Bos, Ganimian y Vegas, 2014a), un insignificante $0,6 \%$ tiene un buen desempeño y el $0,5 \%$, alto. Los resultados subsisten invariablemente desde el 2009.

La figura 2 muestra el desempeño de la competencia científica, sus relaciones contextuales, el género y las brechas sociales y afectivas. En ese sentido, Portugal progresa, mientras que Perú permanece igual; ambos, por debajo de la media de la OCDE. Asumiendo el género, los varones destacan en ciencias (Bos, Ganimian y Vegas, 2014b). Los puntajes de Perú resaltan el limitado aporte de la competencia científica a la competitividad e innovación nacional, sin generar incrementos en el aprendizaje .

En el contexto social, la competencia científica presenta valores diferenciados. En Perú, país en auge económico, no origina mayores impactos; en tanto que, en Portugal, país en aprietos, expresa un repliegue en el impacto. Los resultados evidencian la importancia del saber científico para acortar o cerrar las brechas en el aprendizaje y, proyectarse hacia un mejor desarrollo. Una dinámica que en Latinoamérica se manifiesta económicamente, pero en Perú, no aporta a reducir las desigualdades, sino para ensancharlas más aún, entre estudiantes pobres y ricos (en más de dos años y medio de escolaridad). Al respecto, los estudiantes ricos tienen padres altamente educados, que trabajan en ocupaciones cualificadas, cuentan con más libros en casa y mayores accesos al arte (Bos, Ganimian y Vegas, 2014d). En torno a la resiliencia, el potencial para sobresalir de niveles deficitarios y lograr buen desempeño, en Perú, los resilientes no superan el $1 \%$ y, un amplio sector, tiene menos posibilidades de buenos aprendizajes. Este aspecto permanece inalterable en Portugal.

\section{MATERIAL Y MÉTODOS}

La investigación sobre la ciencia escolar se centró en el análisis de la competencia científica de los currículos de Educación Básica de Perú y Portugal, y el contraste con el Marco de la Evaluación PISA 2012. De ese modo, se describe y compara los componentes curriculares. Se recurrió al análisis documental

Tabla 2. Variables y dimensiones analizadas.

\begin{tabular}{lc}
\hline Estadísticos & Valores \\
\hline A. Organización del tiempo curricular & OTC \\
B. Concepción de la competencia científica & $\mathrm{CCC}$ \\
B.1. Capacidades científicas & $\mathrm{CapCi}$ \\
B.2. Conocimientos científicos & $\mathrm{ConCi}$ \\
B.3. Actitudes Científicas & $\mathrm{ActCi}$ \\
B.4. Contextos Científicos & $\mathrm{ConCi}$ \\
C. Diseño Curricular de la ciencia escolar & DCCE \\
\hline
\end{tabular}

como método de aproximación al objeto. Supuso un conjunto de operaciones propias para la búsqueda del dato relevante, mediante el análisis curricular. En mirada crítica, explica la dinámica y lógica del diseño $y$, permite inferir comprensiones resignificadas, "inferencias reproducibles y válidas que puedan aplicarse a un contexto" (Krippendorff, 1990, p. 28). En tal intención, el análisis de contenido contribuyó objetiva, sistemática, cualitativa y cuantitativamente a la formulación de datos representativos, al aportar sintética y sustantiva a la comprensión de las variables y dimensiones comparadas.

Las variables de análisis permiten comparar la naturaleza prescriptiva del currículo. Propiamente, el tiempo curricular responde a la periodización de las actividades educativas en tiempos semanales y diarios, destinado al aprendizaje científico mediante la organización curricular. La concepción de la competencia científica remite a las formas de aprehenderla, así como a su estructuración y desarrollo curricular. Indican también, presencias y ausencias de los componentes de configuración, así como posturas ideológicas. Los componentes curriculares (capacidades, conocimientos, actitudes y contextos) responden a lo previsto por PISA (OCDE, 2009), en tanto que, el diseño curricular remite al proceso de elaboración plasmado en documentos curriculares.

\section{Fuentes de estudio}

En el desvelamiento de las variables curriculares formuladas, el análisis documental implicó una operación intelectual encauzada a componer un producto derivado de los documentos originales, como respuesta a una secuencia operacional y epistémica. La sistematización valido el proceso de interpretación y análisis. Para el propósito, los documentos curriculares analizados fueron:

Diseño Curricular Nacional de Educación Básica Regular (Ministerio de Educación, 2009) h†tp:// www.minedu.gob.pe/p/ministerio-normatividad.html

Currículo Nacional do Ensino Básico 2001 (Ministério da Educação e Ciência, 2001)http://www.dgidc.minedu.pt/ensinobasico/index. php?s=directorio\&pid $=2$

Marcos y pruebas de evaluación de PISA 2012. Matemáticas, Lectura y Ciencias http://www.oecd. org/centrodemexico/medios/programainterna cionaldeevaluaciondelosaumnos pisa.htm

El análisis se realizó durante el 2015, cuando los currículos se mantenían vigentes en los países. Se usó tres Fichas de observación (Turpo, 2014):

i) Organización del Tiempo Curricular (OTC),

ii) Componentes de la Concepción de la Competencia 
Científica (CCC) y

iii) Componentes del Diseño Curricular (CDC).

\section{RESULTADOS}

\section{Organización escolar en Perú y Portugal}

Los sistemas educativos ordenan la naturaleza del currículo y su tratamiento. Su reconocimiento distingue los substratos que subyacen a la enseñanzaaprendizaje. En lo que respecta a la organización escolar, en el Perú, la obligatoriedad de la educación básica comprende 11 años de escolaridad (de 6 a 17 años de años) y, en Portugal, 9 años (de 6 a 15). La educación secundaria en Perú está organizada en 2 ciclos ( $\mathrm{VI}$, dos grados: 1ㅇ-웅 y VII en tres: 3으, 4으, 5으); mientras que, en Portugal, abarca los 3 últimos grados $(10$ ㅇ, 11 ㅇ 12 으.

En Portugal, durante la educación básica, los estudiantes participan en evaluaciones formativas, globales y de nivel. La evaluación global resume el rendimiento de los objetivos curriculares (metas curriculares) y se efectúa al final del período lectivo y de ciclo. En el I ciclo, la evaluación es descriptiva, en II y III, cuantitativa y descriptiva. Para todos, la promoción está reglada por una evaluación global final (al superar reciben un diploma de enseñanza básica emitido por el órgano administrativo de la escuela). Al término de la escolaridad básica, optan por su continuidad, entre dos tipos diferentes de estudios secundarios: i) formación general y ii) formación profesional (Turpo 2014).

En Perú, se organiza por niveles y modalidades integradas y articuladas a los principios, fines y objetivos educacionales. Está constituida por ciclos, en función a logros de aprendizaje. La obligatoriedad comprende VII ciclos. La perspectiva de continuidad asegura la articulación de las competencias. La promoción de grado en los primeros años de estudio (III ciclo) es automática, subsiguientemente, bimestral o trimestral, mediante evaluaciones formativas. No existe una evaluación final por ciclo.

Un aspecto diferencial de la educación está expresado por la "cultura de la evaluación", una dinámica presente en mayor grado en Portugal que en Perú. Ello, contribuye a una mejor receptividad evaluativa, al preparar para situaciones futuras. Las evaluaciones enfatizan, básicamente, en una medición del rendimiento académico, más que al sentido formativo, al desarrollar habilidades para una mayor efectividad en las pruebas estandarizadas, crean también sesgos sobre las tipologías estudiantiles, en razón a sus rendimientos. En una u otra disposición, la cultura evaluativa propicia cuestionamientos al quehacer educativo.

Otro rasgo distintivo se encuentra en el plan de estudios, un componente revelador de los contrastes de organización curricular. En Portugal se organiza en áreas disciplinares, mientras que en Perú en áreas curriculares (Turpo, 2014). Ambos, prevén ciclos de estudio, aunque con diferente composición, en grados/años de estudio. Los ciclos y grados de estudio que configuran la educación básica en el Perú se distribuyen en áreas curriculares. En Portugal, en una matriz curricular de componentes curriculares (áreas disciplinares, facultativas y electivas). En perspectiva comparada, existen variaciones notables. Perú, en cuanto a las áreas de formación, abarca 11 obligatorias más Tutoría. En Portugal, 7 obligatorias y una opcional, más una oferta de disciplinas complementarias. Situación que en Perú no se presenta, por el contrario, las horas de libre disponibilidad se dirigen a áreas consideradas prioritarias (Matemática, Comunicación y Educación para el Trabajo).

Tabla 3

Organización del tiempo curricular de la competencia científica (Ciclo/s de estudios que evalúa PISA)

\begin{tabular}{|c|c|c|c|c|c|c|}
\hline & \multicolumn{3}{|c|}{ Perù } & \multicolumn{3}{|c|}{ Portugal } \\
\hline & \multicolumn{6}{|c|}{ Periodo Anual } \\
\hline & $36 \mathrm{Ser}$ & anas $\times 5 \mathrm{di}$ & $t=180$ & $35 \mathrm{~S}$ & s. 5 & 175 \\
\hline & \multicolumn{6}{|c|}{ Jornada Horaria/Ciclo } \\
\hline \multirow{4}{*}{ Areas del curriculo } & Perú (Dia & 7 horas/Set & $\operatorname{ana}=35 \mathrm{~h}$ ) & Portugal ( & hora. & in $4=30 \mathrm{~b}$ ) \\
\hline & \multicolumn{3}{|c|}{ Ciclo/Grado de estudio } & \multicolumn{3}{|c|}{ Ciclo/Año de estudio } \\
\hline & VI & \multicolumn{2}{|c|}{ VII } & \multicolumn{3}{|c|}{ III } \\
\hline & $1^{\circ}$ & $2^{\circ}$ & $3^{\circ}$ & $7^{\circ}$ & $8^{\circ}$ & $9^{\circ}$ \\
\hline Ciencia, Tecnologia y Ambiente & $3-4$ & $3-4$ & $3-4$ & - & - & - \\
\hline Cièncias Nahurais/Fisico-Quimicas & - & - & $\because$ & 6 & 6 & 6 \\
\hline Educación para el Trabajo & $2-3$ & $2-3$ & 2.3 & & & \\
\hline Expressões e Tecnologias & & & & 4 & 4 & 3. \\
\hline Total h/semana $\left(1 \mathrm{~h}=45^{\prime}\right)$ & $5-7$ & $5-7$ & $5-7$ & 10 & 10 & 9 \\
\hline Tiempo Curricular Anual & $180-252$ & $180-252$ & $180-252$ & 350 & 350 & 350 \\
\hline$\%$ Tiempo Curricular Anual & \multicolumn{3}{|c|}{$1200(15-21)$} & \multicolumn{3}{|c|}{$1100(31)$} \\
\hline \multicolumn{4}{|c|}{ Diferencia Anual $[(350-315)-(180-252)] \%$} & \multicolumn{3}{|c|}{$98-170(28-51)$} \\
\hline Tiempo Curricular Ciclo ( 3 años) & & $540-756$ & & & 1015 & \\
\hline \multicolumn{4}{|c|}{ Diferencia Ciclo $[1015-(540-756)] \%$} & \multicolumn{3}{|c|}{$475-259(26-47) \%$} \\
\hline
\end{tabular}




\section{Organización del tiempo curricular de la ciencia escolar}

El periodo anual de estudios abarca en Portugal 35 semanas (entre 1 y 5 semanas menos que los europeos), así como jornadas diarias, de 6 horas (entre 1 y 3 horas menor de sus vecinos). En Perú, el año escolar comprende una semana más (36) y una hora adicional en la jornada diaria (7).

En el resumen anual, los estudiantes peruanos estudian entre 180 y 252 horas de ciencias, un 28 a 51 \% menos del tiempo programado para los portugueses (350). En Perú, de las horas anuales obligatorias ( 1200 horas anuales), se destina a su aprendizaje alrededor de un quinto (15-21) \% del año escolar. Un tiempo menor al de las otras áreas evaluadas: Matemática (no menos de 6 horas, $16 \%$ ) y Comunicación (24\%, un mínimo de $8+2$ de ingles). En Portugal, las horas de la enseñanza de la ciencia escolar representan casi $1 / 3$ del tiempo curricular anual, mayor al de Matemática y algo menor en Comunicación. En general, para su aprendizaje, Portugal prevé en el año escolar un mayor número de horas que Perú y una proporción similar durante el ciclo de estudios (Turpo, 2014). Las diferencias expresan los énfasis curriculares previstos para el desarrollo científico. Portugal concentra un mayor tiempo curricular para su enseñanza, un indicador de la importancia de su aprendizaje. Esta previsión de tiempo tendría una incidencia en la diferencia de los resultados.

\section{Concepción de la competencia científica}

La CCC incide en nuestras vidas, por lo que resulta imperativo comprender la organización de sus componentes, así como los aspectos delimitantes y su relación con lo regulado por PISA. Entre Portugal y Perú existe una sustancial diferencia en la concepción de la ciencia escolar, mientras que, en Perú, el enfoque de competencia lleva a una integración de varias disciplinas (física, química, biología) en una única área curricular; en Portugal, cada disciplina conserva su autonomía, e interactúa con áreas afines (tecnologías), de manera interdisciplinaria e interdependientemente. PISA se orienta en esta última dirección, de un abordaje interdisciplinario, antes que integrado.

La complejidad que supone el entendimiento del enfoque de competencias que prioriza PISA, presente en ambos países, Perú recurre a una perspectiva integradora de los conocimientos previos, mientras que Portugal, prioriza una lógica cognitiva, de diferenciación y afirmación del carácter inter-relacional del conocimiento científico. Sea en el sentido de la recuperación o de afirmación del conocimiento escolar, su tratamiento curricular remite a una movilización por el aprendizaje y, a una consiguiente aplicación de los saberes (conocimientos, habilidades, valores, actitudes). El énfasis en una u otra opción, evidencia la necesidad de una identificación y formulación de los saberes, de forma explícita para su aprehensión y aplicación como una competencia que requiere de la apropiación de otros saberes.

La tabla 4 muestra la organización y distribución de los diversos componentes curriculares, y su correlato con lo establecido por PISA.

La concepción sobre la competencia científica en Perú y Portugal difiere en la forma de entender a las capacidades. PISA comprende que éstas posibilitan la aplicación del conocimiento en una determinada situación o contexto, a partir de: a) identificar asuntos o temas científicos, b) explicar científicamente los fenómenos y c) usar la evidencia científica. En ese entender, Perú identifica con b), la comprensión de la información y asume que guarda relación con a) y con c), indagación y experimentación. En tanto que, Portugal, reconoce las capacidades que se presentan como formas de conocimiento $y$, se organizan respecto de PISA, del siguiente modo: a) demandan un conocimiento epistemológico, con b) varios tipos de conocimiento (substantivo, de raciocinio y de comunicación) y, con c) identifica el conocimiento procesual. La diversidad de correspondencias revela la multiplicidad dimensional y su polisemia. Otro aspecto destacable del análisis corresponde a la dimensión del conocimiento, es decir, del conjunto de contenidos de diversa naturaleza (conceptos, destrezas, valores y actitudes) a concretarse en la competencia científica. PISA los organiza en dos tipos: a) contenidos científicos, organizados en sistemas disciplinares (físicos, biológicos, tecnológicos, geológicos y astronómicos) y, b) contenidos sobre las ciencias, de conocimientos sobre investigación científica, explicaciones científicas y relaciones CTS.

En Perú, los conocimientos son definidos desde tres ejes organizadores de la relación Ciencia, Tecnología y Ambiente (CTA). Estos se consolidan mediante una única competencia (competencia científica) y una sola docencia en el área curricular (que presupone un dominio integral de las disciplinas). En ese sentido, los conocimientos se recrean en Ejes Organizadores, tratando de aproximarse a los sistemas planteados por PISA, aunque distantes y en una lógica diferente, es decir, integrados como contenidos y no interrelacionados como disciplinas científicas. Los ejes de conocimiento declarados en el currículo están en relación con: a) la naturaleza de una ciencia integrada, de unidad conceptual de la ciencia, de globalización de conocimientos aparentemente divorciados, que remiten a una visión particular de la ciencia y a una estructura común de tratamiento curricular unitario. En esa perspectiva, los conocimientos demandados en b), se encuentran subsumidos en los Ejes Organizadores y comprendidos bajo un único método científico, definido como la aplicación correcta de la teoría al 
Tabla 4

Componentes de la competencia científica.

\begin{tabular}{|c|c|c|}
\hline $\begin{array}{l}\text { Marcos y pruebas de evaluación } \\
\text { de PISA 2012. Matemáticas, } \\
\text { Lectura y Ciencias (PISA) }\end{array}$ & $\begin{array}{c}\text { Diseño Curricular Nacional de } \\
\text { Educación Básica Regular } 2009 \\
\text { (Perú) }\end{array}$ & $\begin{array}{c}\text { Currículo Nacional do Ensino } \\
\text { Básico 2001 } \\
\text { (Portugal) }\end{array}$ \\
\hline \multicolumn{3}{|c|}{ Capacidades (CapCi) (aplicación del conocimiento a una situación o contexto) } \\
\hline $\begin{array}{l}\text { Identificar asuntos o temas } \\
\text { científicos }\end{array}$ & & Conhecimento epistemológico \\
\hline $\begin{array}{l}\text { Explicar científicamente los } \\
\text { fenómenos }\end{array}$ & Comprensión de la información & $\begin{array}{l}\text { Conhecimento substantivo } \\
\text { Raciocinio } \\
\text { Comunicação }\end{array}$ \\
\hline Usar la evidencia científica & Indagación y experimentación & Conhecimento procesual \\
\hline \multicolumn{3}{|c|}{ Conocimientos (ConCi) (conceptos, destrezas, valores y actitudes } \\
\hline Contenidos de la ciencia & Ejes organizadores & Temas organizadores \\
\hline $\begin{array}{l}\text { a) Sistemas físicos } \\
\text { b) Ciencias de la vida } \\
\text { c) Ciencias de la tierra y del } \\
\text { espacio } \\
\text { d) Sistemas tecnológicos } \\
\text { e) La investigación científica } \\
\text { f) Las explicaciones científicas } \\
\text { g) Las relaciones Ciencia, } \\
\text { Tecnología y Sociedad (CTS) }\end{array}$ & $\begin{array}{l}\text { a) Mundo físico, tecnología y } \\
\text { ambiente } \\
\text { b) Mundo viviente, tecnología y } \\
\text { ambiente } \\
\text { c) Salud integral, tecnología y } \\
\text { sociedad }\end{array}$ & $\begin{array}{l}\text { a) Terra no espaço } \\
\text { b) Terra em transformação } \\
\text { c) Sustentabilidade na terra } \\
\text { d) Viver melhor na terra }\end{array}$ \\
\hline \multicolumn{3}{|c|}{ Actitudes (ActCi) (disposición a usar el conocimiento científico en beneficio personal y social } \\
\hline $\begin{array}{l}\text { a) Sistemas físicos } \\
\text { b) Ciencias de la vida } \\
\text { c) Ciencias de la tierra y del } \\
\text { espacio } \\
\text { d) Sistemas tecnológicos } \\
\text { e) La investigación científica } \\
\text { f) Las explicaciones científicas } \\
\text { g) Las relaciones Ciencia, } \\
\text { Tecnología y Sociedad (CTS) }\end{array}$ & $\begin{array}{l}\text { a) Mundo físico, tecnología y } \\
\text { ambiente } \\
\text { b) Mundo viviente, tecnología y } \\
\text { ambiente } \\
\text { c) Salud integral, tecnología y } \\
\text { sociedad }\end{array}$ & $\begin{array}{l}\text { a) Terra no espaço } \\
\text { b) Terra em transformação } \\
\text { c) Sustentabilidade na terra } \\
\text { d) Viver melhor na terra }\end{array}$ \\
\hline a) Interés en la ciencia & $\begin{array}{l}\text { Curiosidad } \\
\text { Iniciativa e interés } \\
\text { Valoración de la formación }\end{array}$ & $\begin{array}{l}\text { Curiosidade } \\
\text { Perseverança e a seriedade }\end{array}$ \\
\hline $\begin{array}{l}\text { b) Aceptación del pensamiento } \\
\text { científico }\end{array}$ & Valoración del lenguaje científico & $\begin{array}{l}\text { Respeitando e questionando os } \\
\text { resutados } \\
\text { Flexibilidade } \\
\text { Reformulação do seu trabalho } \\
\text { Reflexão crítica }\end{array}$ \\
\hline $\begin{array}{l}\text { c) Sentido de la responsabilidad } \\
\text { hacia el uso de los recursos y } \\
\text { el medio ambiente }\end{array}$ & $\begin{array}{l}\text { Participación } \\
\text { Cuidado del ecosistema } \\
\text { Proposición de alternativas } \\
\text { Valoración de la biodiversidad }\end{array}$ & $\begin{array}{l}\text { Desenvolvimento do sentido } \\
\text { estético } \\
\text { Ética e a sensibilidade } \\
\text { Avaliando o seu impacte }\end{array}$ \\
\hline \multicolumn{3}{|c|}{ Contextos (ConCi) (situaciones de la vida que implican la aplicación de Ciencia y Tecnología) } \\
\hline $\begin{array}{l}\text { Interés personal, que afectan a la } \\
\text { persona, a sus amigos, } \\
\text { compañeros o familiares }\end{array}$ & $\begin{array}{l}\text { Promoción y manifestación } \\
\text { de la curiosidad, exploración y } \\
\text { reflexión de procesos científicos }\end{array}$ & $\begin{array}{l}\text { Compreenderem o mundo em } \\
\text { que vivem, com as suas múltiplas } \\
\text { interacções }\end{array}$ \\
\hline $\begin{array}{l}\text { Interés social que tienen que ver } \\
\text { con la comunidad en la que se vive }\end{array}$ & $\begin{array}{l}\text { Comprensión de la naturaleza } \\
\text { a partir de la indagación y la } \\
\text { investigación }\end{array}$ & $\begin{array}{l}\text { Experiências educativas } \\
\text { conducentesaodesenvolvimento } \\
\text { de competências de natureza } \\
\text { diversa }\end{array}$ \\
\hline $\begin{array}{l}\text { Interés global por afectar al } \\
\text { mundo entero }\end{array}$ & $\begin{array}{l}\text { Construcción reflexiva de } \\
\text { conocimientos acerca de las } \\
\text { interaccioneseinterdependencias } \\
\text { sociales, ecológicas y geográficas } \\
\text { que ocurren en el contexto }\end{array}$ & $\begin{array}{l}\text { Experiências educativas que } \\
\text { procuram integrar vários } \\
\text { aspectos inerentes quer ao } \\
\text { ensino, quer à aprendizagem dos } \\
\text { alunos em ciências }\end{array}$ \\
\hline
\end{tabular}


aprendizaje, lo que conlleva a un indoctrinamiento excesivo, sin mayor aporte a la comprensión de la naturaleza de la ciencia.

En Portugal, los contenidos de la competencia científica se organizan en Temas Organizadores, acorde a la interrelación Ciencia, Tecnología, Sociedad y Ambiente (CTSA). Estas, se desagregan en competencias específicas (metas curriculares). Evidencian así, el recorrido y balance sistemático de los aprendizajes articulados en ciclos. Los conocimientos se organizan en temas interdisciplinares $y$, en un régimen de codocencia (docente por disciplina). Desde esa perspectiva, conjugan las disciplinas, a partir de subtemas que revelan la naturaleza articular de la ciencia. La organización prevista asigna coherencia conceptual y metodológica y, una perspectiva holística y sistemática "para alcanzar fines pretendidos siguiendo una línea y secuencia" (Roldão, 2003, p. 28). De ese modo, los temas organizadores coinciden en mayor grado con la organización sistémica promovido por PISA.

Perú y Portugal, en vertiente comparativa, distan en la organización del conocimiento científico. Perú transita en una lógica de integración conceptual y, Portugal, en interrelación disciplinar. Este último tipo de organización está más próximo a lo de PISA. En cualquier caso, está "no excluye, pero exige, la apropiación sólida y amplia de contenidos, organizados en una síntesis integradora, apropiada por el sujeto, permitiéndole 'convocar' ese conocimiento con respecto a las diferentes situaciones y contextos" (Roldão, 2003, p. 24).

En correspondencia a las actitudes científicas, de la disposición para usar el conocimiento científico en benéfico personal o social, PISA los organiza en tres dimensiones (ver tabla 4). En esa línea, en el currículo peruano, las actitudes relacionadas con a) se desarrollan desde la curiosidad, iniciativa e interés y la valoración formativa; con b), coinciden en la estimación del lenguaje científico y, con c), desde la participación, cuidado del ecosistema y valoración de la diversidad. Las primeras actitudes guardan relación con el favorecimiento del aprendizaje y a una mayor implicación científica. No solo como vocación de estudio y de formación profesional, sino como continuidad formativa. Este grupo de actitudes se relaciona con la responsabilidad científica ambiental, preocupación central del currículo peruano, enfatizado en el enfoque asumido (CTA) (Turpo, 2016). En tanto que, las actitudes en el currículo de ciencias de Portugal, difieren en distintos grados, en ciertas dimensiones de la competencia científica, del énfasis puesto en Perú. Coinciden en a), en buena sintonía, con b), resaltan otras, además de las consideradas en Perú (reflexión crítica, flexibilidad, respeto y cuestionamiento a resultados y reformulación). Las últimas, complementan la valoración del lenguaje científico. Con c), las discrepancias son evidentes, por el énfasis del enfoque CTSA. En perspectiva comparada, no sólo transcienden la consideración del impacto científico en el ambiente, además, consideran el sentido ético y estético de los problemas sociales, al disponer el uso de la ciencia en beneficio personal y social, soslayada en el currículo peruano.

Respecto a los contextos de la competencia científica de PISA, es decir, las condiciones que movilizan las capacidades, conocimientos y actitudes para aprender y emprender en ciencias, son apreciables los contrastes, más en Perú que en Portugal. En este último país, se presentan en adecuada relación con los referentes, aunque no se evalúa directamente, más si su pertenencia como factores que pueden mejorar el interés por el aprendizaje. Es claro que el contexto permite explicitar los conceptos en relación con el enfoque, como recurso de un abordaje adecuado de los conocimientos científicos, tanto para: 1) la consecución de una cultura científica para todos (finalidad generalista), como 2) el aprendizaje de los fundamentos básicos de quienes opten por un desempeño más trascendente (finalidad especializada). Las tensiones generadas entre las finalidades requieren de estrategias curriculares que las conjuguen. En esa intención, Perú resalta una diversidad de procesos reflexivos para la aprehensión de la competencia científica, Portugal se centra más en la diversidad de experiencias que conduzcan a su concreción (Turpo, 2016). Esta distinción revela el carácter incidental del contexto en la adquisición de la competencia científica, Portugal se orienta en un sentido más aplicativo, de experimentación e interrelación con el medio; mientras que Perú, opta por procesos comprensivos, fundados en la exploración y reflexión científica.

En síntesis, la diversidad representativa de los componentes de la competencia científica de Perú y Portugal, responden a alineaciones de diversa magnitud a lo normado por PISA. Éstas, no se presentan como contradicciones, sino como respuesta a las singularidades sociales y como efectos del enfoque subrayado. En Perú, en las capacidades y conocimientos predomina la integración de saberes (ciencia integrada) y un énfasis del conductismo sobre el constructivismo (Turpo-Gebera, 2013). En tanto que, en Portugal, respecto a las capacidades y conocimientos, prima un carácter de interrelación de las disciplinas científicas (interrelación disciplinar) y una enseñanza basada y orientada por un positivismo lógico (Bispo, Delourdes, Pereira y Vázquez, 2013). En cuanto a las actitudes, los acercamientos son variables, al igual que los contextos.

\section{Diseño curricular de la ciencia escolar}

El DCCE proporciona una serie de procedimientos, de componentes curriculares que sustentan la programación, para satisfacer las demandas 


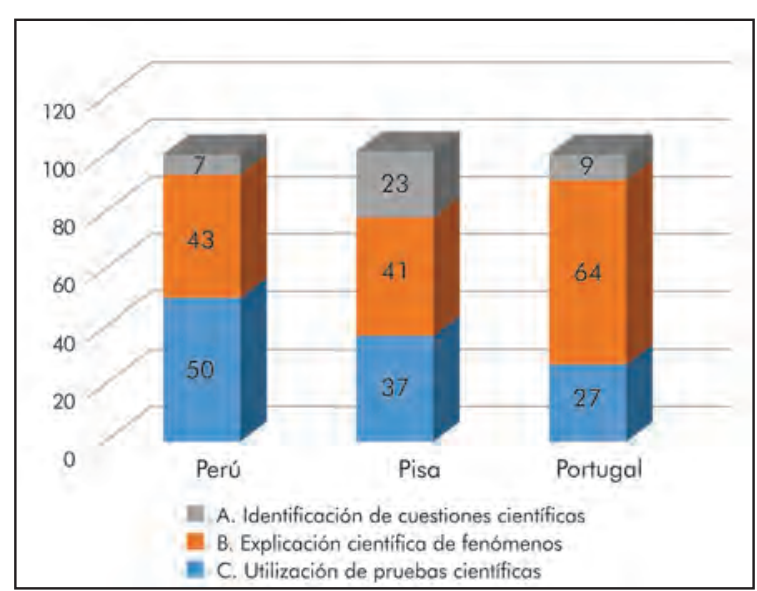

Figura 3. Comparación de capacidades de la competencia científica en el grado/año, con respecto a PISA 2012 (\%).

educativas. En primera instancia, se presenta los resultados en el grado de estudios (VII Ciclo $=3$ ㅇ y 4 응 Grado en Perú y III Ciclo = 9으o en Portugal) de los estudiantes evaluados por PISA.

La capacidad contrastada de la competencia científica hace evidente la relevancia de B (explicación científica de fenómenos), menor en Perú, y superada ampliamente por Portugal, en más de 50\%. El énfasis justificaría, en alguna medida, la diferencia de resultados en la prueba PISA 2012, favorable a Portugal. Es importante tener en cuenta que, en materia educativa, es previsible una concurrencia de factores más que una predominante.

Si se compara la previsión de las capacidades para el desarrollo de las competencias científicas durante el ciclo de estudios que evalúa PISA (III ciclo en Portugal y VI-VII en Perú), estas difieren aún más en el análisis global.

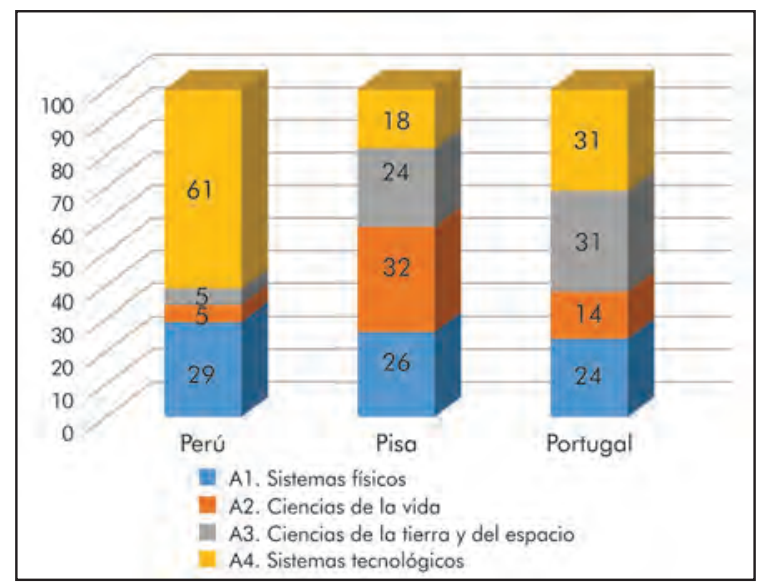

Figura 5.Comparación de la competencia científica en el grado/año correspondiente a PISA $2012(\%)$.

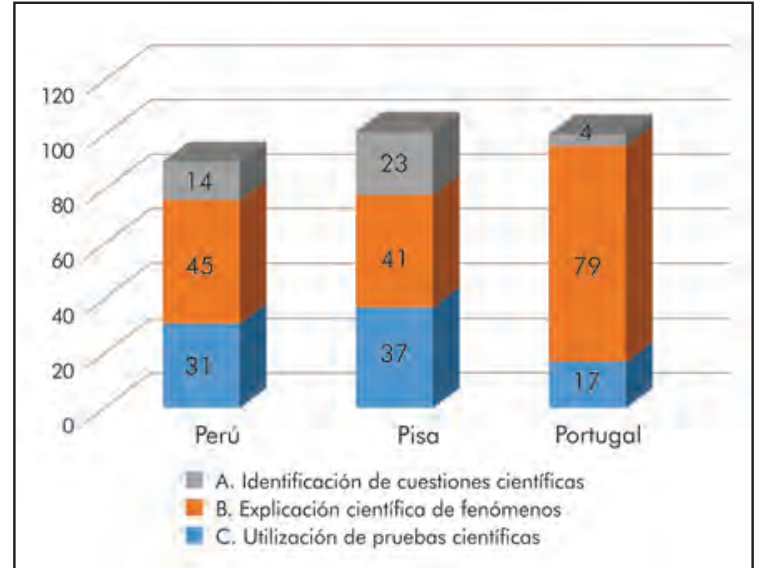

Figura 4. Comparación de capacidades de la competencia científica del ciclo de estudios previos a la prueba PISA 2012 (\%).

La figura 2 señala la importancia asignada a las capacidades científicas, en grados diversos, pero con énfasis semejantes. Durante los estudios previos a la evaluación PISA, se contempla sesgos disimiles en los currículos. La explicación científica de fenómenos (B) es asumida en buena medida en los currículos. Hay que considerar que la capacidad de explicación constituye una de las operaciones esenciales de la ciencia, al dotar de una mayor capacidad y significatividad a los estudiantes en la resolución de problemas de interés. A través de esta capacidad se hurga en las razones de los hechos científicos, mediante enunciados verdaderos sobre los procesos y productos y no de modo distinto (Klimovsky, 1995).

Otro de los componentes analizados está dado por el conocimiento a adquirir durante el proceso construido en la escuela (ciencia escolar), como parte de la competencia científica.

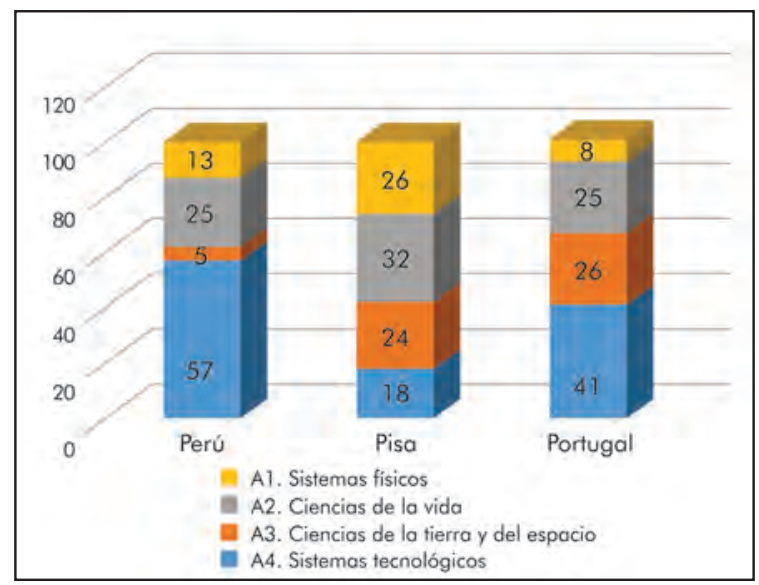

Figura 6.Comparación de conocimientos de la competencia científica en el ciclo previo a PISA 2012 (\%). 


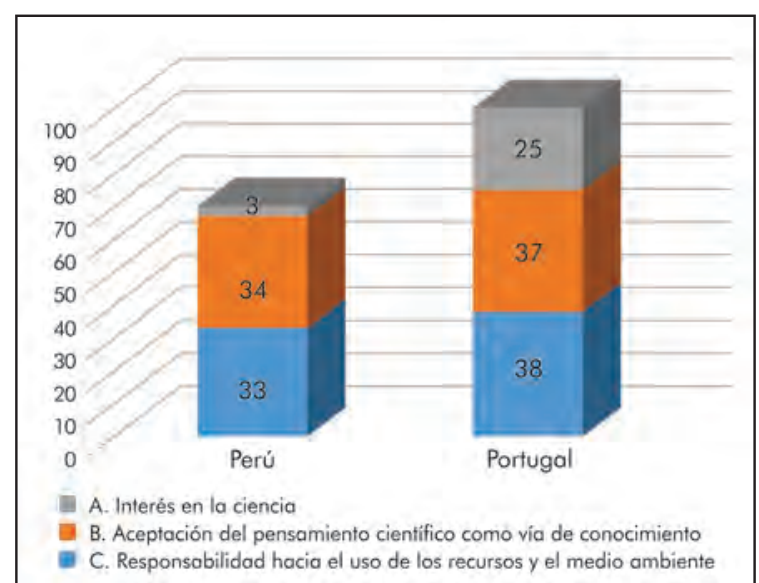

Figura 7:Comparación de las actitudes de la competencia científica en PISA 2012 (\%).

Este saber trasciende las explicaciones cotidianas, no es un conocimiento científico en sí, sino una elaboración ajustada a las características escolares. Propiamente, son conocimientos que se aprenden, como substrato para repensar las relaciones en su construcción.

Los currículos analizados muestran diferencias en los conocimientos o contenidos escolares respecto a lo establecido por PISA. Así, respecto del sistema A1 (Sistemas físicos), la de menor variabilidad en los sistemas, Portugal presenta un $2 \%$ de proporción menor a lo establecido, mientras que Perú supera a ambos, en un $2 \%$ y $5 \%$. En los demás sistemas de conocimientos se presentan amplios contrastes. En A2 (Ciencias de la vida), Portugal prevé contenidos en un $50 \%$ menos de PISA, y Perú en 6 veces menor. En A3 (Ciencias de la tierra y del espacio), el currículo peruano dista 5 veces de PISA, en tanto que Portugal supera en un $7 \%$. El mayor contraste se advierte en A4 (Sistemas tecnológicos), pues Perú concentra una mayor proporción, más de 3 veces que PISA; mientras que Portugal está próximo a duplicarlo. Los efectos revelan las diferencias en el año/grado específico que evalúa PISA. Se observa la dinámica en el ciclo de estudios previo (Perú, VI y VII, Portugal, III) en la figura 6.

La distribución de los conocimientos científicos en el ciclo previo a la prueba PISA, revela el énfasis de ambos en el sistema A4. En Perú más que Portugal y superior a la previsión establecida por PISA. En A2, Perú supera tenuemente a Portugal, en más de $6 \%$ de PISA. En relación al A3, Portugal se encuentra levemente por encima; mientras que Perú, muy por debajo (4 veces). Sobre el sistema A1, ambos currículos están por debajo de lo de PISA, Portugal más distante que Perú. Como se comprueba, la distribución del conocimiento científico en los currículos de ciencias sigue patrones diferenciados, tanto a nivel del año/grado evaluado como en el ciclo previo. En cualquier caso, en ninguna de las situaciones se aproximan a los referenciales establecidos por PISA. Cada currículo conserva sus propias particularidades y con diferencias apreciables en los resultados de la evaluación PISA 2012.

Respecto a las actitudes previstas en los currículos de la competencia científica, éstas, se manifiestan en diversos matices, con resultados discrepantes de los componentes cognitivos (A). La comparación invita a considerar la necesidad de impulsar de manera independiente los conocimientos (Kozlow y Nay, 1976), a fin de que el desarrollo actitudinal siga una línea propia. De ahí, el diseño de dominios específicos sobre las actitudes científicas. En ese sentido, la organización curricular de las actitudes hacia la competencia científica en Perú y Portugal siguen una dinámica con ligeros contrastes.

\section{DISCUSIÓN}

En los currículos de ciencias estudiados, las discrepancias de organización de los componentes de la competencia científica reflejan los énfasis, no sólo de la concepción del enfoque dominante sobre las relaciones de la ciencia y tecnología, CTA en Perú y CTSA en Portugal, sino también de las formas de integración de conocimientos. Las formas de construcción curricular conllevan en Perú, a propuestas de integración conceptual, con una dinámica sustentada en una aparente disolución de los contenidos de las diversas disciplinas científicas en una única intención de aproximación a las ciencias (ciencia integrada), y con el ejercicio de un régimen de docencia única. En Portugal, se contraponen a la integración curricular, y siguen una vía disciplinar, de identidad de la materia científica en el aprendizaje de las ciencias y en la perspectiva de una co-docencia. La contrastación curricular de la competencia científica "pone de manifiesto el alcance, dirección y magnitud de las influencias exógenas sobre los cambios curriculares nacionales" (Astiz, 2014, p. 8), y al mismo tiempo, hace evidente las diferencias incubadas localmente.

Los contextos y circunstancias que contribuyen al desarrollo de la ciencia escolar posibilitan inferir una cierta geopolítica del conocimiento científico, determinado por el lugar que éstos ocupan en el currículo. En ese sentido, se privilegia la inclusión de determinados contenidos curriculares que marquen una diferenciación de poder, y creen una ilusión de saber en la periferia. Una trampa de la modernidad, de "que el conocimiento es des-incorporado y deslocalizado y que es necesario, desde todas las regiones del planeta, "subir" a la epistemología de la modernidad" (Walsh, 2003, p. 3). Se trata de que en los currículos escolares se priorizan unos tipos de conocimientos disciplinares sobre otros. Por ejemplo, las ciencias de la vida y de la tierra y del espacio en Portugal, por sobre los sistemas físicos en Perú. La perspectiva planteada sobre los énfasis y supresiones 
de determinados conocimientos en el currículo supone una visión del mundo (colonialista), donde el poder trasnacional (OCDE, BM, FMl, etc.), dirige, expande, orienta e impone políticas de generación o reproducción de conocimientos. De este modo, se aplaca la insumisión y se impone reformas curriculares acordes a "las leyes del mercado, el individualismo y la economía del conocimiento" (Salas, 2013, p. 12). Este último mecanismo, enfatiza en la priorización y/o exclusión de unos conocimientos en los currículos escolares, con el pretexto de lograr mayor eficiencia y eficacia de los procesos productivos y un aparente desarrollo socioeconómico.

Resulta también criticable, la afirmación mayoritaria de un tipo de capacidad científica: la explicación científica de los fenómenos por sobre la identificación y utilización. Las variaciones curriculares distan de lo referenciado por PISA, más en Perú que en Portugal. Este último país, presenta un mayor alineamiento curricular a lo establecido por la OCDE, a través de PISA, expresado en la prevalencia de dicha capacidad, reconocida como fundamental en el quehacer científico, y que es escasamente abordada en el currículo peruano.

La organización del tiempo curricular constituye otro de los aspectos diferenciadores, de los periodos y asignaciones temporales para la competencia científica. En Portugal se asignan más tiempos que Perú al aprendizaje de las ciencias. Una dinámica reveladora del grado de importancia concedida a las ciencias en las sociedades analizadas (Turpo, 2014). El tiempo curricular en el desarrollo de la competencia científica es fundamental, a fin de concretar los aprendizajes que conformen una masa crítica de estudiantes comprometidos con el aprendizaje de las ciencias. En ese sentido, los currículos de las ciencias deben posibilitar: 1) el acceso a la cultura científica para ser partícipe de sus implicaciones sociales y 2) una disposición para hacer de la ciencia una actividad de desarrollo profesional futuro.

Con la intención de construir un currículo acorde a las necesidades locales, pero con mirada global, el currículo debe recuperar la historicidad de las ciencias en cada realidad, para que contribuya de modo particular y en interrelación con los saberes generales al desarrollo nacional. El proceso, demanda superar la obsesión curricular por las oportunidades y el empoderamiento individual como únicas fuentes de progreso personal como social (Astiz, 2014). Una decisión que no debe substraer a los gobiernos de sus responsabilidades identitarias, sino por el contrario, afirmarlas. En ese orden de precisiones, PISA es un medio para acercar al país a la realidad mundial, pero no el único ni inevitable referente. Tampoco cabe atribuirle más significatividad que la informativa, de un valor de medición entre otros, para juzgar los avances y retrasos experimentados por los sistemas educativos de cada país.

En mirada comparativa, los resultados diferenciados de la prueba PISA 2012, entre Perú y Portugal, devienen de la organización curricular, si bien no es el único factor, su significatividad como hallazgo no es despreciable. Esto, a pesar de que PISA sostenga que no evalúa contenidos, sino aplicaciones a situaciones cotidianas. Una posibilidad de explicación discurriría en comprender, teóricamente, que el desarrollo de una determinada competencia depende de lo establecido en el diseño curricular, de la transcripción de las experiencias de enseñanza y aprendizaje en la escuela y, por consiguiente, en el aula. Una aspiración no siempre concretada o parcialmente implementada.

Como se ha visto, en el análisis del currículo de la ciencia escolar, PISA establece de manera normativa lo enseñable, señalando un decurso de aprendizaje, a través de la instrumentalización de un enfoque curricular que prescribe los contenidos que considera importantes. Por ende, su intervención responde a intenciones de gobernanza de los sistemas educativos. En esa pretensión, dispone de un tipo de organización curricular que homogeniza a los sistemas educativos. Recuérdese que el currículo no en un dispositivo neutro, sino un traductor de las finalidades socioeducativas privilegiadas, dirigido a formar en las convenciones sociales, rituales, costumbres y significados del mundo circundante, sin mayores cuestionamientos.

Los factores estudiados desvelan las distintas configuraciones para organizar el currículo, cuyas diferenciaciones se aprecian en los resultados de PISA. Las discrepancias que no parecen ser apreciadas por PISA, instituyen unos lineamientos e intervenciones evaluativas uniformes. Tales advertencias "corroborarían la hipótesis del arraigo de un modelo de estandarización (o normalización) mundial del curriculum escolar" (Astiz, 2014, p. 6), al establecer unos únicos referentes evaluativos, contribuye a invalidar las diferencias y a fundar unos prototipos o patrones educativos, que privilegien determinados saberes en desmedro de otros, que podrían ser relevantes y significativos para las sociedades que lo asuman.

No resulta desconocido que la evaluación PISA es objeto de múltiples controversias. Además de las críticas en torno a la representatividad de la muestra, o de comparaciones entre realidades tan disimiles, se resalta en este análisis, la imposición de unos criterios de organización curricular que soslayan la realidad local donde se gestan los aprendizajes, al establecer un currículo generalizado y derivado de un mismo paradigma (Kamens, 1992). Un patrón de difusión cultural que sortea las características históricas y geográficas nacionales. La tendencia a la 
homogenización revela una intencionalidad de control de los sistemas educativos nacionales, de privilegiar un único enfoque educativo: el de las competencias, como único e indispensable dispositivo pedagógico, y al que deben alinearse los países.

La decisión de los países de participar en las evaluaciones PISA, debería obedecer a su carácter informativo. En esa disposición, los sistemas educativos optarían entre adaptarse a las recomendaciones de PISA u optar por un modelo educativo propio, sin caer en subordinaciones. La decisión implica reconocer que cuanto más se enaltece la relación entre la ciudadanía global y local, emerge con mayor énfasis, la valoración de la diversidad autóctona (Meyer y Ramírez, 2010). Es decir, se trata de recuperar en mayor grado, la información de las evaluaciones nacionales, como referente inmediato para el debate curricular; a fin de evadir las exigencias de dominio de unos saberes específicos -conocimientos, habilidades, valores, actitudes-, que "obliguen" a una aplicación intrascendente y descontextualizada a la realidad local (Coll y Martín, 2006). En ese sentido, resulta fundamental, avanzar en la construcción de un currículo propio y "auténtico", lo que no descarta explorar las diferencias con otras realidades, por el contrario, constituye el medio para acercarse en mayor grado a las acciones que orienten la construcción de un diseño curricular pertinente y significativo. Un proceso devenido del debate y del consenso social, y de respuesta a las exigencias del mundo globalizado. Donde se asuma lo local y la centralidad del estudiante en su socialización global, en un mundo de incertidumbres.

\section{REFERENCIAS BIBLIOGRÁFICAS}

Astiz, M. (2014). El Curriculum Escolar y su Abordaje desde la Teoría de la Sociedad Mundial: Revisión y Prospectiva. Archivos Analíticos de Políticas Educativas, 22(25), 1-18. http://dx.doi. org/10.14507/epaa.v22n25.2014

Baker, D. (2011). El efecto educación en el desarrollo social: intelectual y políticamente subestimado. Revista Peruana de Investigación Educativa, 3, 74 95.

Baker, D. y LeTendre, G. (2005). Nacional Differences, Global Similarities. World Culture and the Future of Schooling. California: Stanford University Press.

Bispo, D., Delourdes, M.; Pereira, R. y Vázquez, A. (2013). Alfabetização científica sob o enfoque da ciência, tecnologia e sociedade: implicações para a formação inicial e continuada de profesores. Revista Electrónica de Enseñanza de las Ciencias, 12(2), 313-333. http://reec.uvigo.es/volumenes/ volumen 12/REEC_12_2_5_ex649.pdf
Bos, M., Ganimian, A. y Vegas, E. (2014a). América Latina en 2012. Brief \#3: ¿Cuántos estudiantes tienen bajo desempeño? Washington: DC, BIDOCDE.

Bos, M., Ganimian, A. y Vegas, E. (2014b). América Latina en 2012. Brief \# 4: ¿Cuántos estudiantes logran un desempeño destacado? Washington, DC, BID-OCDE.

Bos, M., Ganimian, A. y Vegas, E. (2014c). América Latina en 2012. Brief \#2: ¿ Cuánto mejoró la región? Washington, DC, BID-OCDE.

Bos, M., Ganimian, A. y Vegas, E. (2014d). América Latina en 2012. Brief \#6: ¿ Cómo se desempeñan los estudiantes pobres y ricos? Washington, DC, BID-OCDE.

Coll, C. y Martin, E. (2006). Vigencia del debate curricular. Revista PRELAC, 3, 6-27.

Espinoza, G. y Torreblanca, A. (2003). Cómo rinden los estudiantes peruanos en comunicación y matemática. Resultados de la Evaluación Nacional 2001. Informe descriptivo. Lima: MED-UMC.

Gil, D y Martínez, J. (2005). ¿̇ara qué y cómo evaluar? La evaluación como instrumento de regulación y mejora del proceso de enseñanza/aprendizaje, en Gil, D., Macedo, B., Martínez, J., Sigfredo, C., Valdés, P. y Vilches, A. (eds.). ¿ Cómo promover el interés por la cultura científica? Una propuesta didáctica fundamentada para la educación científica de jóvenes de 15 a 18 años. (159-182). Santiago, OREALC/UNESCO.

Hargreaves, D. (2005). Personalising Learning 3: Learning to Learn \& the New Technologies. London: Specialist Schools Trust.

Jones, P. (2007). Education and world order. Comparative Education, 43(3), 325-337.

Jonnaert, P., Barrette, J. Masciotra, D. y Yaya, M. (2008). Revisión de la competencia como organizadora de los programas de formación: hacia un desempeño competente. Ginebra: OIE/BIE/UNESCO.

Kamens, D. (1992). Variant forms: Cases with distinct curricula. In J. Meyer, D. Kamens, A. Benavot, Y. Cha \& S. Wong (Eds.). School Knowledge for the Masses: World Models and National Primary Curricular Categories in the Twentieth Century (7484). Washington, DC: The Falmer Press.

Klimovsky, G. (1995). Las desventuras del conocimiento científico. Una introducción a la epistemología. Buenos Aires: AZ Editora.

Kozlow J. \& Nay, M. (1976). An approach to measuring scientific attitudes. Science Education, 60, 147-172.

Krippendorff, K. (1990). Metodología del análisis de contenido. Teoría y Práctica. Barcelona: Paidós Ibérica.

Lawn, M. (2011). Standardizing the European education policy space. European Educational Research Journal, 10(2), 259-272.

Leibfried, S. (2009). PISA: internacionalización de la política educativa o żcómo se llega de la política nacional a la OCDE? Profesorado. Revista de 
Curriculum y Formación del Profesorado, 13(2). http://www.ugr.es/ recfpro/rev132ART9.pdf

Meyer, H. \& Benavot, A. (2013). PISA, power and policy: the appear of global governance of education. Oxford (UK): Symposium Books.

Meyer, J. \& Ramírez, F. (2010). La institucionalización mundial de la educación, en J. Meyer y F. Ramírez (Eds.). La educación en la sociedad mundial. Teoría institucional y agenda de investigación de los sistemas educativos contemporáneos (111-134). Barcelona: Octaedro.

Ministério da Educação e Ciência (MEC). (2014). Portugal. Primeiros resultados PISA 2012. Lisboa: MEC-OCDE.

Ministerio de Educación (MED). (2009). Diseño Curricular Nacional de Educación Básica Regular. Lima: MED.

Organización para la Cooperación y el Desarrollo Económico (OCDE) (2004). Marcos teóricos de PISA 2003: la medida de los conocimientos y destrezas en matemáticas, lectura, ciencias y resolución de problemas. Madrid: MEC, INECSE.

Organización para la Cooperación y el Desarrollo Económico (OCDE) (2005). La definición y selección de competencias clave. Resumen ejecutivo. México, DF.: USAID-OCDE.

Organización para la Cooperación y el Desarrollo Económico (OCDE) (2009). El programa PISA de la OCDE qué es y para qué sirve. Madrid: Santillana.

Organización para la Cooperación y el Desarrollo Económico (OCDE) (2012). Marcos y pruebas de evaluación de PISA 2012. Matemáticas, Lectura y Ciencias. Madrid: MEC-OCDE.

Organización para la Cooperación y el Desarrollo Económico (OCDE) (2006). PISA 2006. Marco de la evaluación. Conocimientos y habilidades en Ciencias, Matemáticas y Lectura. París: OCDE.

Pereyra, M., Kotthoff, H. y Cowen, R. (2013). PISA a examen: cambiando el conocimiento, cambiando las pruebas y cambiando las escuelas. Introducción al monográfico. Profesorado. Revista de Currículum y Formación de Profesorado, 17(2), 6-14.

Programa de la Naciones Unidades para el Desarrollo (PNUD). (2013). Informe sobre Desarrollo Humano 2013. El ascenso del Sur: Progreso humano en un mundo diverso. New York, PNUD.

Roldão, M. (2003). Gestão do currículo e avaliação de competências. As questões dos professores, Lisboa: Presença.
Rychen, D. \& L. Salganik, (2000) (eds.). Defining and selecting key competencies. Göttingen: Hogrefe \& Huber, 2000.

Rychen, D. \& L. Salganik (2003) (eds.). Key competencies for successful life and a well-functioning society. Göttingen: Hogrefe \& Huber.

Salas, L. (2013). Geopolítica del Conocimiento y la Recolonización Educativa. Contexto \& Educação, 89, 12-40.

Schumpeter, J. (2008). The Theory of Economic Development: An Inquiry into Profits, Capital, Credit, Interest and the Business Cycle. New Brunswick (USA) \& London (UK): Transaction Publishers.

Tröler, D. (2009). Armonizar el globo educativo. Política mundial, rasgos culturales y los desafíos a la investigación educativa. Profesorado. Revista de Curriculum y Formación del Profesorado, 13(2), http://www.ugr.es/ recfpro/rev132ART9.pdf

Turpo-Gebera, O. (2013). Posicionamiento de los docentes de ciencias en la evaluación de los aprendizajes: una aproximación a sus subjetividades. Educación Química, 24(2), 230236.

Turpo Gebera, O. (2014). La competencia científica en Perú y Portugal, ante los resultados PISA2012: un análisis comparado de sus diseños curriculares. (Informe de Investigación Posdoctoral). Universidade de Coimbra, Portugal.

Turpo-Gebera, O. (2016). El currículo de la competencia científica en Perú y Portugal. Comuni@cción, 7(2), 15-26.

Unidad de Medición de la Calidad Educativa (UMC) (2014). PISA 2012. Primeros resultados. Informe nacional del Perú. Lima: MED.

Valladares, L. (2011). Las competencias en la educación científica. Tensiones desde el pragmatismo epistemológico. Perfiles Educativos, XXXIII(132), 158-182.

Walsh, C. (2003). Las geopolíticas del conocimiento y colonialidad del poder. Entrevista a Walter Mignolo, Polis, 4. DOI: http://10.4000/polis.7138

Yus, R., Fernández, M., Gallardo, M., Barquín, M., Sepúlveda, M. y Serván, J. (2013). La competencia científica y su evaluación. Análisis de las pruebas estandarizadas de PISA. Revista de Educación, $360,557-576$. 